\title{
THE ENERGY RELEASE-STELLAR ANGULAR MOMENTUM INDEPENDENCE IN ROTATING COMPACT STARS UNDERGOING FIRST-ORDER PHASE TRANSITIONS
}

\author{
M. BEJGER ${ }^{1,2}$, J. L. ZDUNIK ${ }^{2}$, P. HAENSEL ${ }^{2}$ and E. GOURGOULHON ${ }^{1}$ \\ ${ }^{1}$ LUTh, Observatoire de Paris, CNRS, Université Paris Diderot, 5 Place Jules Janssen, 92190 \\ Meudon, France \\ ${ }^{2}$ N. Copernicus Astronomical Center, Polish Academy of Sciences, Bartycka 18, PL-00-716 \\ Warszawa, Poland \\ bejger@camk.edu.pl,jlz@camk.edu.pl,haensel@camk.edu.pl,Eric.Gourgoulhon@obspm.fr
}

\begin{abstract}
We present the general relativistic calculation of the energy release associated with a first order phase transition (PT) at the center of a rotating neutron star (NS). The energy release, $E_{\text {rel }}$, is equal to the difference in mass-energies between the initial (normal) phase configuration and the final configuration containing a superdense matter core, assuming constant total baryon number and the angular momentum. The calculations are performed with the use of precise pseudo-spectral 2-D numerical code; the polytropic equations of state (EOS) as well as realistic EOSs (Skyrme interactions, Mean Field Theory kaon condensate) are used. The results are obtained for a broad range of metastability of initial configuration and size of the new superdense phase core in the final configuration. For a fixed "overpressure", $\delta \bar{P}$, defined as the relative excess of central pressure of a collapsing metastable star over the pressure of the equilibrium first-order $\mathrm{PT}$, the energy release up to numerical accuracy does not depend on the stellar angular momentum and coincides with that for nonrotating stars with the same $\delta \bar{P}$. When the equatorial radius of the superdense phase core is much smaller than the equatorial radius of the star, analytical expressions for the $E_{\text {rel }}$ can be obtained: $E_{\text {rel }}$ is proportional to $(\delta \bar{P})^{2.5}$ for small $\delta \bar{P}$. At higher $\delta \bar{P}$, the results of 1 -D calculations of $E_{\mathrm{rel}}(\delta \bar{P})$ for non-rotating stars reproduce with very high precision exact $2-\mathrm{D}$ results for fast-rotating stars. The energy release-angular momentum independence for a given overpressure holds also for the so-called "strong" PTs (that destabilise the star against the axi-symmetric perturbations), as well as for PTs with "jumping" over the energy barrier.
\end{abstract}

Keywords: dense matter - equation of state - stars: neutron - stars: rotation

\section{Introduction}

Many theories of dense matter predict that at some density larger than the nuclear saturation density, a phase transition (PT) to some "exotic" state (i.e. boson condensate or quark deconfinement) occurs; for review see e.g., [1-3]). A first-order PTs are particularly interesting from the astrophysical and observational point of view, because are associated with a meta-stable state of dense matter. One can thus expect, in the case of PTs occurring in the interior of NSs, the release of non-negligible amount of energy. Here we will focus on the basic features of the 

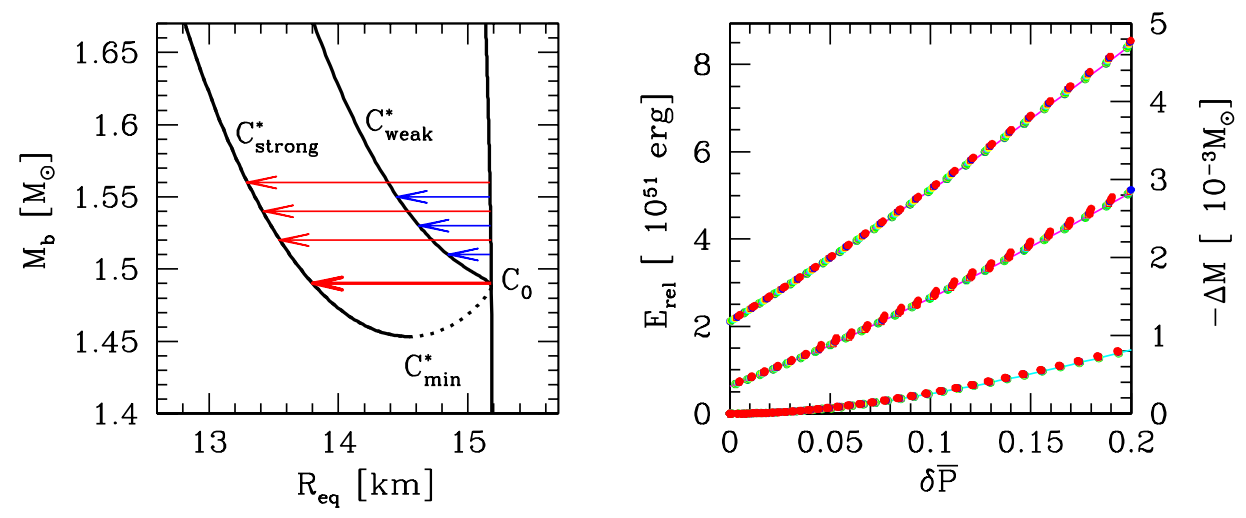

Fig. 1. Left panel: Strong and weak PTs on mass-radius diagram (dotted line marks unstable configurations). Right panel: the energy release $E_{\text {rel }}$ vs the over-pressure parameter $\delta \bar{P}$ for strong (two upper curves) and weak (lowest curve) PTs. Points are colored differently for different total angular momenta $J=(0,0.1, \ldots, 1.3) \times G M_{\odot}^{2} / c$ and follow the curve for the $J=0$ (non-rotating) configurations.

energy release-angular momentum independence; for complete description we refer the reader to $[4,5]$. The text is arranged as follows: in Sect. 2 we briefly describe the results of calculations. Sect. 3 contains conclusions and open questions.

\section{Calculation of the energy release}

The hydrostatic, axi-symmetric and rigidly rotating configurations of compact stars with and without PTs were obtained using the numerical GR library LORENE (http://www.lorene.obspm.fr). We assume that the baryon mass $M_{\mathrm{b}}$ and the total angular momentum $J$ are constant during the PT from the metastable configuration $\mathcal{C}$ to stable configuration $\mathcal{C}^{*}$. From the microscopic point of view the first-order $\mathrm{PT}$ is characterised by the over-pressure parameter $\delta \bar{P}=\left(P_{c}-P_{0}\right) / P_{0}$, where $P_{c}$ is the central pressure of the configuration $\mathcal{C}$ and $P_{0}$ is the equilibrium pressure, at which the PT occurs. We distinguish between the so-called weak and strong PTs, characterised by the density-jump parameter $\lambda=\rho^{*} / \rho$, where $\rho$ and $\rho^{*}$ are the densities of normal and condensed phase at $P_{0}$. PTs with $\lambda>\frac{3}{2}\left(1+P_{0} / \rho c^{2}\right)$ (strong PTs) destabilise the stars with arbitrarily small cores against the axi-symmetric perturbations [6-8]. The energy release (energy difference) is defined as

$$
E_{\mathrm{rel}}=c^{2}\left[M(\mathcal{C})-M\left(\mathcal{C}^{*}\right)\right]_{M_{\mathrm{b}}, J}
$$

and it is quite remarkable that (up to numerical accuracy) it does not depend on the rotation state of the configuration i.e. on the total angular momentum $J$ of the star and agrees well with the $E_{\text {rel }}$ for non-rotating stars. In Fig. 1 we present the results for the polytropes (parametric EOSs in the form of $P=K n^{\Gamma}$, where $n$ is the number baryon density, $K$ is is the pressure coefficient and $\Gamma$ is called the adiabatic index; detailed description of parameters used can be found in [5]). 

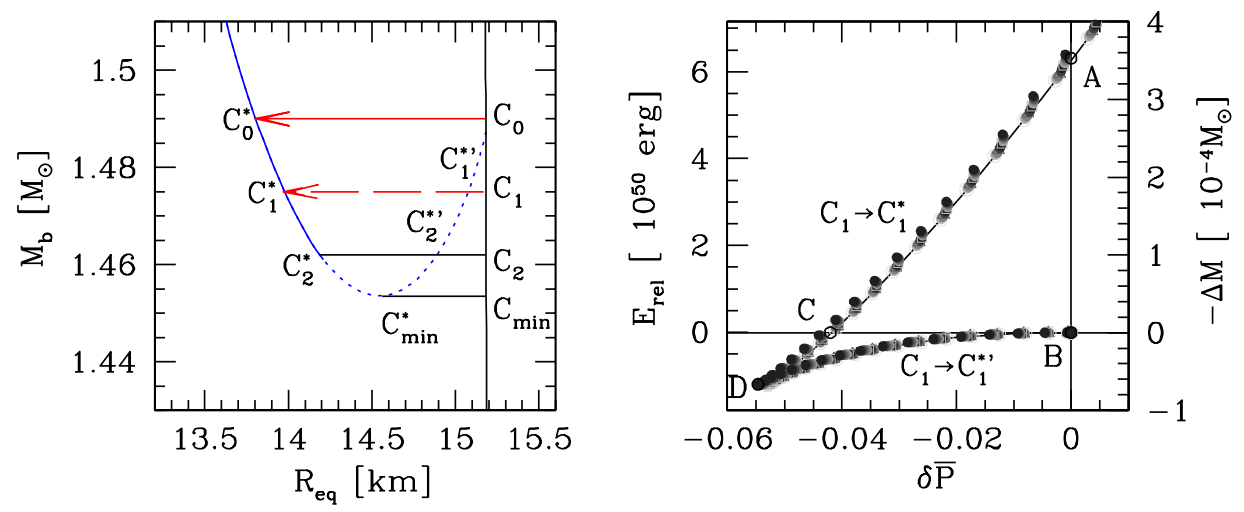

Fig. 2. Left panel: The strong PT with the "jumping" over the energy barrier on the mass-radius diagram. Right: Energy release $E_{\text {rel }}$ vs the over-pressure parameter $\delta \bar{P}$ for the strong PT. Points with different colors correspond to PTs with different angular momentum $J=(0,0.1, \ldots, 0.9) \times$ $G M_{\odot}^{2} / c^{2}$ also for negative $\delta \bar{P}$. Solid line - result for $J=0$ (non-rotating stars). Point A corresponds to $\mathcal{C}_{0} \longrightarrow \mathcal{C}_{0}^{\star}$, point $\mathrm{B}$ to $\mathcal{C}_{0} \longrightarrow \mathcal{C}_{0}^{\star \prime}=\mathcal{C}_{0}$, point $\mathrm{C}$ to $\mathcal{C}_{2} \longrightarrow \mathcal{C}_{2}^{\star}$ and point $\mathrm{D}$ to $\mathcal{C}_{\min } \longrightarrow \mathcal{C}_{\min }^{\star}$.

In the case of realistic EOSs the results are qualitatively the same. We have checked the phenomenon for different realistic EOSs i.e. Skyrme interactions or the kaon condensate, presented as an example here. The crust obeys the EOS of Douchin \& Haensel [9]. The matter below the PT is described using the relativistic meanfield theory, [10]. The dense phase is the kaon condensate, with coupling of kaons to nucleons proposed by Glendenning \& Schaffner-Bielich [11], the optical potential $U_{\mathrm{K}}^{\text {lin }}$ being equal to $-115 \mathrm{MeV}$. The stability of configurations with $P_{c}$ below that for the equilibrium PT is particularly interesting. If the star is excited initially, e.g. is pulsating, then the formation of a large dense phase core is possible, but it requires climbing ("jumping") over the energy barrier associated with formation of a small core. The results are shown in Fig. 2 ,

\section{Conclusions and open questions}

Our numerical calculations show the $E_{\text {rel }}(J)$ independence during the weak as well as strong first-order PTs, for large rotation rates and large oblatnesses of the stars: it is therefore not an property of slow rotating stars only. The independence holds also for PTs with negative over-pressure $\delta \bar{P}$ i.e. when the configuration "jumps" over the energy barrier to reach another stable configuration. For small positive $\delta \bar{P}$ analytical relations were found: $E_{\text {rel }} \propto(\delta \bar{P})^{2.5}$. The energy release $E_{\text {rel }} \sim 10^{51}-10^{52}$ erg is an absolute upper bound on the energy which can released in such PT. In astrophysical situation, the energy can be distributed between stellar pulsations, gravitational radiation, heating of stellar interior, X-ray emission from the neutron star surface, and even a gamma-ray burst.

Currently there is no mathematical proof of the $J$-independence of $E_{\text {rel }}$. It may 
be interesting to look at the problem from the "thermodynamical" point of view. We write the total energy of the star (gravitational mass $M$ ) as

$$
M=\frac{\mu}{u^{t}} A+2 \Omega J+2 \int_{\Sigma_{t}} P N \sqrt{\gamma} d^{3} x,
$$

where $\mu$ is the baryon chemical potential, $u^{t}$ the time component of the fluid 4velocity, $A$ the number of baryons in the star, $\Omega$ the angular velocity, $P$ the fluid pressure, $N$ the lapse function and $\sqrt{\gamma} d^{3} x$ the covariant volume element in the constant $t$ hypersurface $\Sigma_{t}$. Eq. (2) is valid for any axi-symmetric stationary and rigidly rotating fluid star which obeys a barotropic EOS, as established by Bardeen $\&$ Wagoner in 1971 [12]. Since $\mathcal{C}$ and $\mathcal{C}^{*}$ have the same baryon number $A$ and the same angular momentum $J$, we get the energy release

$$
E_{\text {rel }}=\Delta E=A \Delta\left(\frac{\mu}{u^{t}}\right)+2 J \Delta \Omega+2\left[\int_{\mathcal{C}} P N \sqrt{\gamma} d^{3} x-\int_{\mathcal{C}^{*}} P N \sqrt{\gamma} d^{3} x\right],
$$

with

$$
\Delta\left(\frac{\mu}{u^{t}}\right):=\left.\frac{\mu}{u^{t}}\right|_{\mathcal{C}}-\left.\frac{\mu}{u^{t}}\right|_{\mathcal{C}^{*}}, \quad \Delta \Omega:=\left.\Omega\right|_{\mathcal{C}}-\left.\Omega\right|_{\mathcal{C}^{*}} .
$$

The terms on the right-hand-side of Eq. 3 are of comparable magnitude in their contribution to the energy release, so the possible "smallness" of some of them in relation to the others is not responsible for the $E_{\text {rel }}(J)$ independence. We will address the problem of mathematically proving this property in the near future.

\section{Acknowledgements}

This work was partially supported by the Polish MNiI grant no. 1P03D.008.27, MNiSW grant no. N203.006.32/0450 and by the LEA Astro-PF programme. MB was also supported by the Marie Curie Fellowship no. MEIF-CT-2005-023644.

\section{References}

[1] P. Hansel, A. Y. Potekhin and D. G. Yakovlev, Neutron Stars 1 : Equation of State and Structure (New York, USA: Springer, 2007).

[2] N. K. Glendenning, Compact stars: Nuclear physics, particle physics, and general relativity (New York, USA: Springer, 1997).

[3] F. Weber, Pulsars as astrophysical laboratories for nuclear and particle physics (IoP Publishing, Bristol \& Philadelphia, 1999).

[4] J. L. Zdunik, M. Bejger, P. Haensel and E. Gourgoulhon, Astron. Astrophys. 465, $533(2007)$.

[5] J. L. Zdunik, M. Bejger, P. Haensel and E. Gourgoulhon, ArXiv e-prints 707 (2007).

[6] Z. F. Seidov, Soviet Astronomy 15, 347 (1971).

[7] B. Kaempfer, Phys. Lett. B 101, 366 (1981).

[8] J. L. Zdunik, P. Haensel and R. Schaeffer, Astron. Astrophys. 172, 95 (1987).

[9] F. Douchin and P. Haensel, Astron. Astrophys. 380, 151 (2001).

[10] J. Zimanyi and S. A. Moszkowski, Phys. Rev. C 42, 1416 (1990).

[11] N. K. Glendenning and J. Schaffner-Bielich, Phys. Rev. C 60, 025803 (1999).

[12] J. M. Bardeen and R. V. Wagoner, Astrophys. J. 167, 359 (1971). 